\title{
EXPERIMENTAL ASSESSMENT OF PTFE POST-ARC ABLATION
}

\author{
Y. Babou*, P. Corfdir, R.-P. Suetterlin \\ Corporate Research, ABB Switzerland Ltd., Segelhofstrasse 1K, CH-5405 Baden-Dättwil, Switzerland \\ * yacine.babou@ch.abb.com
}

\begin{abstract}
The study addresses the post arc ablation (PAA) of PTFE (Polytetrafluoroethylene) material after being stressed by high current arcs. Arcs were generated in ambient air applying AC or DC current profiles to reach energy input in the range $7-22 \mathrm{~kJ}$. The characterization has been performed essentially based on standard optical measurement techniques. The shadowgraph technique enabled us to show that the PAA flow is composed by a significant amount of carbonaceous soots lasting for several dozens of milliseconds after current interruption. The pyrometry technique allowed to estimate the soots temperature in the range 1400-2200 K.
\end{abstract}

Keywords: post arc ablation, pyrometry, shadowgraphy.

\section{Introduction}

The understanding of the ablation process of PTFE (Polytetrafluoroethylene) material undergoing intense incident heat fluxes is of critical importance for the design and optimization of various systems such as space propulsion systems $[1,2]$, thermal protection systems for atmospheric entries [3, 4] and ablationassisted current interruption systems $[5,6]$. In this later case, the ablation of the PTFE material interacting with high current arcs needs to be determined with enough accuracy in order to enable reliable prediction of the interrupting performances. Especially in the case of high voltage circuit breakers based on self-blast approach [7], the knowledge of the PTFE ablative behavior after current interruption remains of considerable importance since it critically affects the arc breakdown voltage during the hot dielectric recovery phase. So far, the post-arc ablation (PAA) of the PTFE has been poorly investigated while it drives significantly the flow properties (its composition and pressure distributions) and consequently the hot dielectric recovery performances. The experimental investigations presented hereafter are aimed at characterizing the PTFE PAA after being stressed by high current arcs.

\section{Experimental setup}

The experiments have consisted of generating high current arcs in a tube made of grey PTFE to assess the post-arc ablation phenomenon in ambient air. We have used PTFE cylindrical tubes of $45 \mathrm{~mm}$ length with an internal diameter of $7 \mathrm{~mm}$ (see Figure 1). The PTFE tubes were tested by applying either AC or DC current profile in order to assess the effect of the current steepness (transition from peak value to zero) on the PAA. The arc was ignited by means of a copper ignition wire. The generic experimental setup adopted for assessing PTFE PAA is sketched in Figure 2.

The setup includes a capacitor bank able to store energy up to $240 \mathrm{~kJ}$. To achieve DC current profiles,
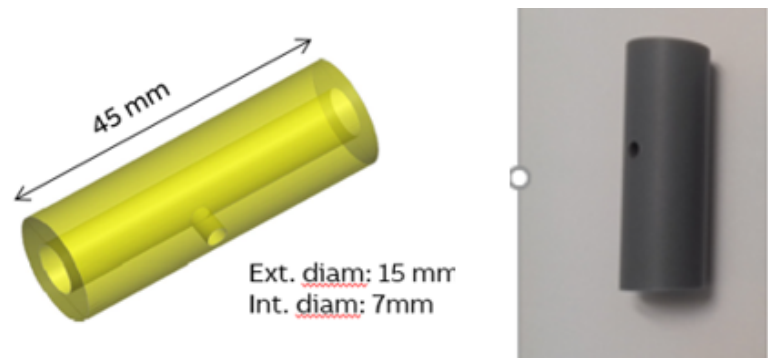

Figure 1. Tested PTFE tubes. (Left) Tube design. (Right) Photograph of actual tube.

we have used an interleaved Buck converter with three parallel lines as power supply that allows one to generate an arbitrary current profiles with an intensity of up to $2 \mathrm{kA}$ per line, resulting in a total maximum current of $6 \mathrm{kA}$. In order to provide the desired current intensity, the voltage measured between the electrodes as well as the actual current intensity are used to compute the switching times of insulated gate bipolar transistors (ABB HiPak IGBTs) located at each of the three lines of the converter. Using three parallel paths with specific offsets in the switching phase makes it possible to achieve a considerable reduction in the current ripple. A thyristor is mounted in parallel to the test object for fast current switching ( $<20 \mu \mathrm{s})$. To obtain AC pulses, the capacitor bank is discharged through an inductance and the arc. The values of the capacitance and inductance are selected to result in a roughly $10 \mathrm{~ms}$ sine half-wave, corresponding to a $50 \mathrm{~Hz}$ grid frequency. Note that for all AC experiments, the thyristor remains connected to the test object but is not triggered. The arc voltage has been measured with a regular differential voltage probe and the current flowing through the test object has been measured by means of a Rogowski probe.

The diagnostic setup comprises a shadowgraph system, a two-colors pyrometer and a high-speed camera for direct visualization during the arc phase. For this 


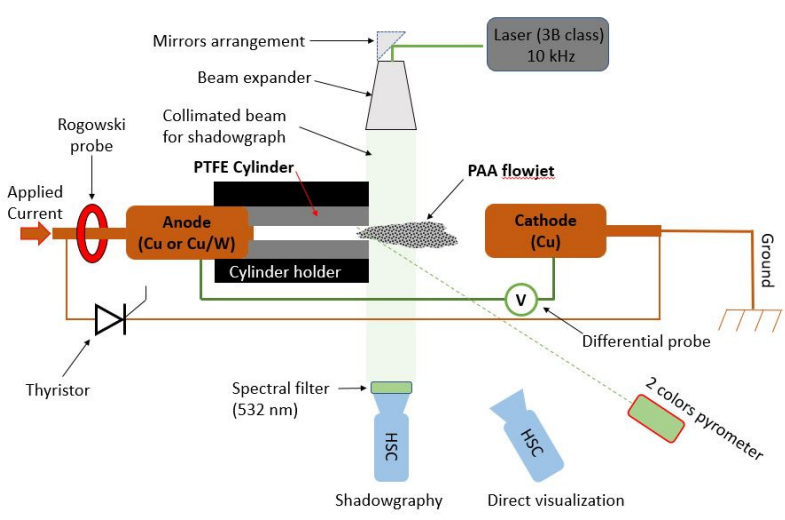

Figure 2. Sketch of the experimental setup.

camera, a 1.6 neutral density filter was mounted on the objective lens and the frame rate was set to $41 \mathrm{kHz}$ with an exposure time of $0.9 \mu \mathrm{s}$. The shadowgraphy system combines a frequency doubled Nd:YAG laser, emitting at $532 \mathrm{~nm}$ and operated at $10 \mathrm{kHz}$, with a high-speed camera (HSC) to record shadowgrams during and after arcing. A neutral density filter providing an optical density of 2.6 and a bandpass filter centered at $532 \mathrm{~nm}$ are mounted on the objective lens of the $\mathrm{HSC}$, and the exposure time was set to $1 \mu \mathrm{s}$. Before the test campaign, we tuned finely the shadowgraph setup, using a supersonic jet of cold air as object of reference to adjust the camera settings to obtain images of highest quality. The two-colors pyrometer is equipped with $\mathrm{Si}$ and Ge detectors, measuring infrared light intensity in the spectral ranges $0.9-1.04 \mu \mathrm{m}$ and $1.1-1.8 \mu \mathrm{m}$, respectively. The pyrometer is coupled with an optical fiber which was arranged to collect the emission from the inner surface of the PTFE tube. Note that this goal can be reached only for an exhaust flow which is optically transparent in the spectral range detected by the pyrometer while the actual exhaust flow after the current interruption is composed of dense stream of solid particles as we will see. As a consequence the pyrometer measures the temperature of that stream of particle.

\section{Results}

Typical shadowgraph records taken in the post-arc phase after the application of $2 \mathrm{kA} \mathrm{DC}$ current for $10 \mathrm{~ms}$ are shown in Figure 3. The displayed situation is fairly representative of the overall considered test conditions. After current zero, a directional jet of small particles is streaming from the PTFE tube lasting for several tens of milliseconds. The fact that this jet appears black stems to the fact that it is opaque at the laser wavelength $(532 \mathrm{~nm})$. With time after current zero, the aggregates of bigger size are released and gradually the stream of particles slows down and its dynamics is dominated by diffusion transport, characterized by a strong mixing with ambient air. A visual inspection of the PTFE tube and electrodes after a single test reveals the presence of large amount of black powder (see Figure 4). The composition of the powder has been analyzed by means of $\mathrm{X}$ rays spectroscopy and a typical image is reported in Figure 5. It turned out that this powder consists in its larger extent of carbonaceous soots (of about $100 \mathrm{~nm}$ size) with traces of oxygen and fluor and in its lesser extent of large copper clusters (of few micrometers size) resulting from the anode erosion.
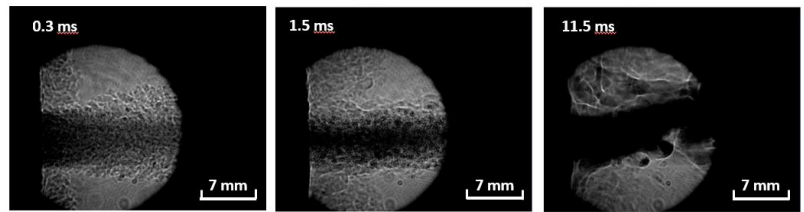

Figure 3. Typical shadowgraphs records at different time after current zero.
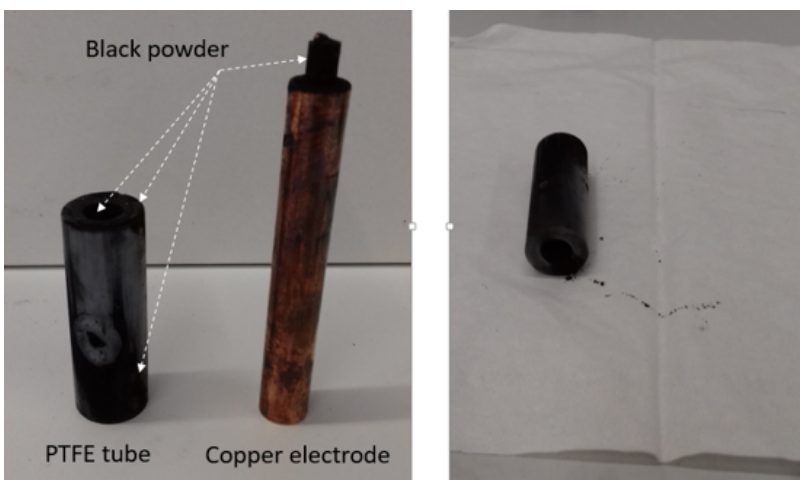

Figure 4. Post test visual inspection.

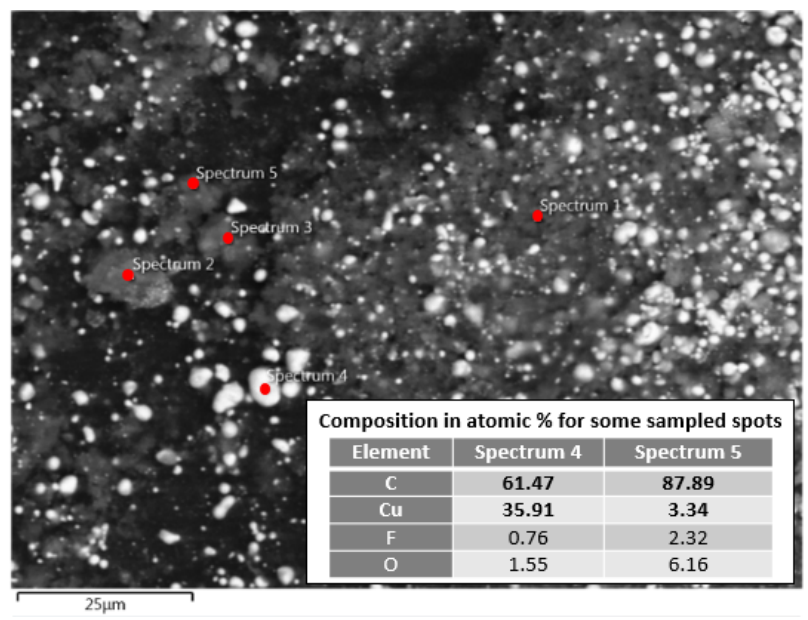

Figure 5. Energy dispersive X-ray spectroscopy mapping of a sample of black powder. The white spots correspond to the copper agreggates. The grey background corresponds to the carbonaceous soot. (In insert) Typical composition of the black powder.

\subsection{DC current tests}

In a first step, we investigate the PAA following on the application for $10 \mathrm{~ms}$ of a DC current pulse of amplitude ranging from 1.5 to $3 \mathrm{kA}$, see Table 1 . The 


\begin{tabular}{lllll}
\hline \hline Current (kA) & $\mathbf{1 . 5}$ & $\mathbf{2 . 0}$ & $\mathbf{2 . 5}$ & $\mathbf{3 . 0}$ \\
\hline Energy (kJ) & 7.5 & 11.4 & 15.1 & 20.2 \\
\hline \hline
\end{tabular}

Table 1. DC test series settings.
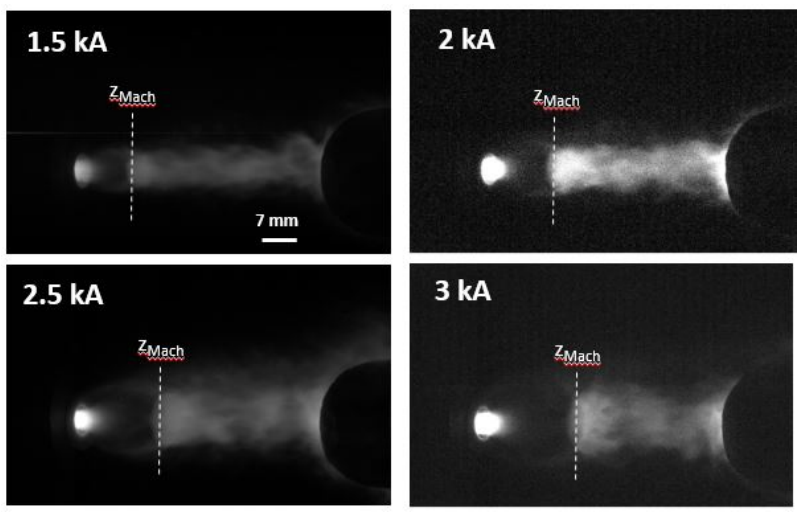

Figure 6. Mach disk position for the DC test series.

corresponding energy inputs were determined on the basis of the arc voltage measurements.

The pressure built-up in the tube during the arc phase has been estimated on the basis of the HSC images taken during the arc phase. The exhaust flow exhibits the typical structure of a supersonic jet characterized by stationary shockwave patterns with the presence of so-called Mach disk as seen in Figure 6. With increasing current intensity, the position of the first Mach disk $\left(z_{\mathrm{M}}\right)$ with respect to the tube exhaust increases, indicating that the pressure within the PTFE tube increases as well. From the Mach disk position, the total pressure in the tube is estimated using the empirical relation reported in [8] for ideal gas expansion. Although such estimate in our conditions is debatable, it gives a fair indication of the pressure evolution with applied current, see Table 2 .

\begin{tabular}{lllll}
\hline \hline Current (kA) & $\mathbf{1 . 5}$ & $\mathbf{2 . 0}$ & $\mathbf{2 . 5}$ & $\mathbf{3 . 0}$ \\
\hline$z_{\mathrm{M}}(\mathrm{mm})$ & 9 & 12 & 15 & 21 \\
Pressure (bar) & 3.7 & 6.5 & 10.2 & 20 \\
\hline \hline
\end{tabular}

Table 2. Estimates of the tube pressure during DC arcing phase.

The effect of the applied DC current on the PAA can be appreciated from the shadowgraph records reported in Figure 7 for $1.2 \mathrm{~ms}$ after current interruption. With increasing applied current (i.e. with increasing energy input), the diameter of the PAA jet tends to broaden, the jet of particles appears to be denser and lasts for longer times.

The temperature time profiles rebuilt from the pyrometer measurements in the post-arc phase are displayed in Figure 8. The measurements start from about $1 \mathrm{~ms}$ after current interruption because the pyrometer was operated during the arc phase and
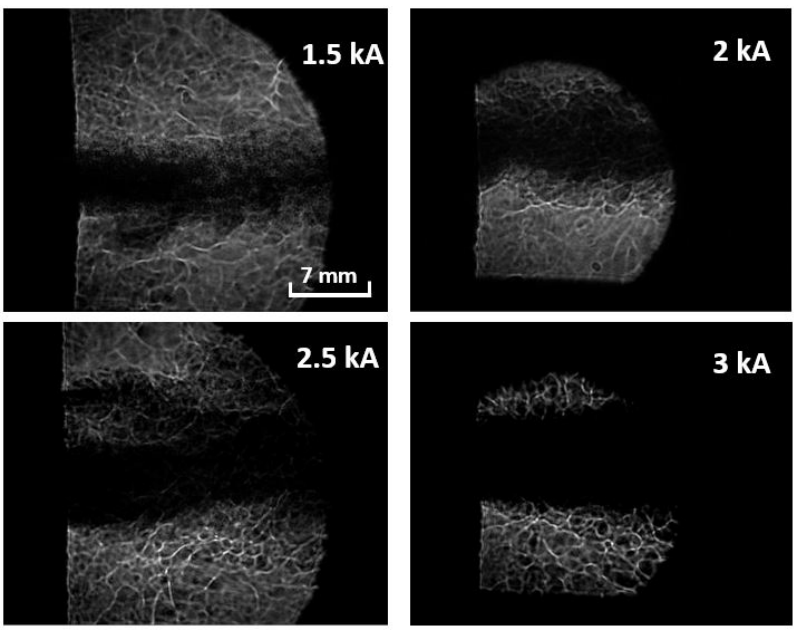

Figure 7. Shadowgraph records for the DC test series taken about $1 \mathrm{~ms}$ after current interruption.

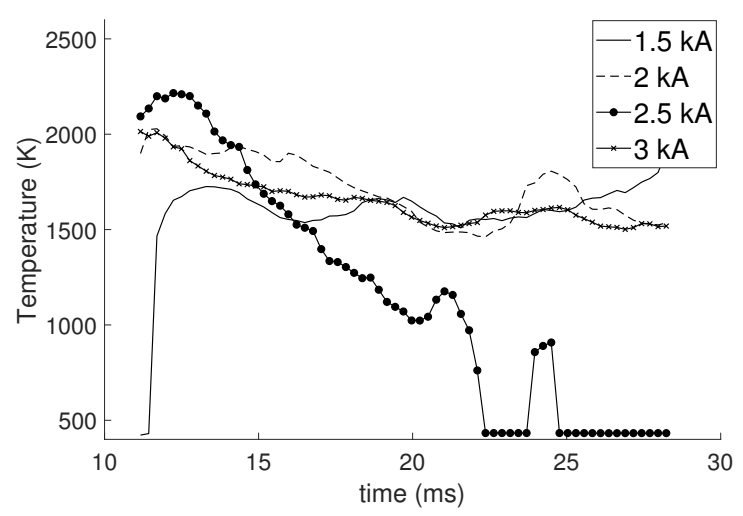

Figure 8. Temperature profiles for the DC test series.

saturated by the arc jet light. It took about $1 \mathrm{~ms}$ to go out from saturation. The evolution of the temperatures does not follow a net trend but exhibits instead a strongly irregular plot because the stream of particle itself exhibits a fluctuating dynamics characterized by a kind of intermittent or "bursty"behavior. This make difficult to interpret the trends followed by the temperature with increasing current and to draw a definitive statement. But still one can note that the temperatures lie in the range $1500-2200 \mathrm{~K}$ for several milliseconds. Above $20 \mathrm{~ms}$ after the current injection, the signal is close to the noise level therefore the rebuilt temperature profiles are no more reliable.

\subsection{AC current tests}

In a second step, we investigate the post arc ablation following on the application for $10 \mathrm{~ms}$ of a $50 \mathrm{~Hz} \mathrm{AC}$ current profile. For this test series, the peak values of the applied current pulses are set to achieve pulse energies comparable to that of the DC experiments, see Table 3. The effect of the applied AC current on the PAA flow is shown in Figure 9 for $1 \mathrm{~ms}$ after current interruption. The overall situation is similar to the DC test series: increasing the applied current leads to a more prominent stream of particle due 


\begin{tabular}{lllll}
\hline \hline Current peak (kA) & $\mathbf{2 . 3}$ & $\mathbf{3 . 1}$ & $\mathbf{3 . 8}$ & $\mathbf{4 . 5}$ \\
\hline Energy (kJ) & 8.6 & 14 & 18.2 & 22.4 \\
\hline \hline
\end{tabular}

Table 3. AC test series setting.
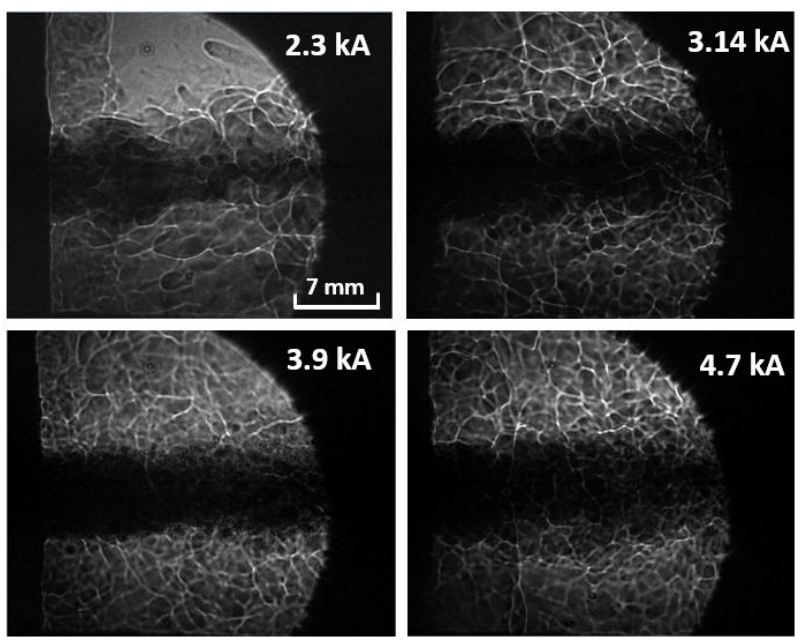

Figure 9. Shadowgraph records for the AC test series taken about 1 ms after current interruption.

to a more intense PAA process. The temperature profiles rebuilt from the pyrometer records also exhibit similar features to the DC tests and cover the same temperature range. However, for the AC test series, the profiles seem to pass by a plateau or a weak local minimum just after current interruption, before steadily decreasing. While for the DC test series, one could say that, in the main, the temperature decays without passing by a plateau. Further tests are required to confirm the observations and assess possible reasons for temperature differences depending on the applied current profiles.

\section{Conclusions}

The experiments reported here enabled us to characterize the PAA phenomenon taking place after stressing a PTFE material with high current arcs in ambient air. As a matter of fact, the PAA is characterized by the synthesis of a significant amount of carbonaceous soots also containing a non-negligible amount of oxygen and fluor. The shadowgraphy in combination with pyrometry technique were found to be well-suited to characterize the PAA flow main features. In our test conditions, the PAA flow consists of a stream of particles lasting for several tens of milliseconds and of temperature in the range $1400-2200 \mathrm{~K}$. The time evolution of the temperature seems to be affected by the applied current profile (either AC or DC) however supplementary investigations are required to substantiate this point. Beyond such initial assessment of the PAA, by combining these measurements in the frame of appropriate assumptions, it is also possible to infer the ablation mass rate of PTFE against the input

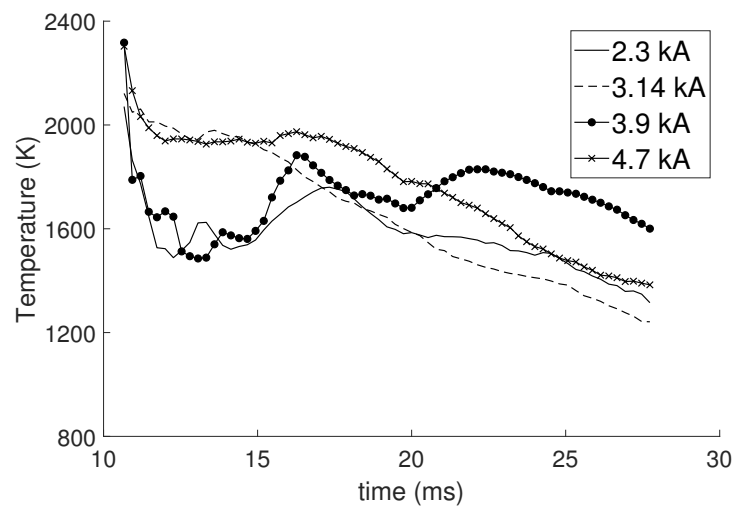

Figure 10. Temperature profiles for the AC test series.

energy. Ultimately it should be mentioned that in actual circuit breaker arrangement, the PTFE made nozzle throat is typically cleared by colder gas blown from the heating or compression volumes, that would modify the PAA characteristics with respect to the present results.

\section{Acknowledgements}

The authors acknowledge Martin Seeger for his fruitful inputs and discussion and Maud Martinon for performing the analysis of the soot sample.

\section{References}

[1] W. J. Guman and P. E. Peko. Solid-propellant pulsed plasma microthruster studies. Journal of Spacecraft and Rockets, 5(6):732-733, 1968. doi:10.2514/3.29340.

[2] Y. Chan, C. Montag, G. Herdrich, and T. Schönherr. Review of thermal pulsed plasma thruster-Design, characterization, and application. In Proc. 34th Int. Electr. Propuls. Conf., pages 1-10, 2015.

[3] N. Diaconis, J. B. Fanucci, and G. W. Sutton. The heat protection potential of several ablation materials for satellite and ballistic re-entry into the earth's atmosphere. Planetary and Space Science, 4:463-478, 1961. doi:10.1016/0032-0633(61)90152-0.

[4] J. G. Marvin and R. B. Pope. Laminar convective heating and ablation in the Mars atmosphere. AIAA Journal, 5(2):240-248, 1967. doi:10.2514/3.3948.

[5] H. Taxt. Ablation-assisted current interruption in medium voltage switchgear-Development and prospect. In Proceedings of the Nordic Insulation Symposium, volume 24, 2015. doi:10.5324/nordis.v0i24.2281.

[6] M. Seeger. Perspectives on research on high voltage gas circuit breakers. Plasma Chemistry and Plasma Processing, 35(3):527-541, 2015. doi:10.1007/s11090-014-9595-4.

[7] M. Seeger, J. Tepper, T. Christen, and J. Abrahamson. Experimental study on PTFE ablation in high voltage circuit-breakers. Journal of Physics D: Applied Physics, 39(23):5016, 2006. doi:10.1088/0022-3727/39/23/018.

[8] H. Ashkenas and F. S. Sherman. Structure and utilization of supersonic free jets in low density wind tunnels, 1965. Technical Report NASA-CR-60423. 